This item was submitted to Loughborough's Research Repository by the author.

Items in Figshare are protected by copyright, with all rights reserved, unless otherwise indicated.

\title{
Individually modifiable risk factors to ameliorate cognitive aging: a systematic review and meta-analysis
}

PLEASE CITE THE PUBLISHED VERSION

http://dx.doi.org/10.3109/13697137.2015.1078106

\section{PUBLISHER}

Taylor \& Francis (@ International Menopause Society)

\section{VERSION}

AM (Accepted Manuscript)

\section{PUBLISHER STATEMENT}

This work is made available according to the conditions of the Creative Commons Attribution-NonCommercialNoDerivatives 4.0 International (CC BY-NC-ND 4.0) licence. Full details of this licence are available at: https://creativecommons.org/licenses/by-nc-nd/4.0/

\section{LICENCE}

CC BY-NC-ND 4.0

\section{REPOSITORY RECORD}

Lehert, Philippe, Paulina Villaseca, Eef Hogervorst, Pauline M. Maki, and Victor W. Henderson. 2019. "Individually Modifiable Risk Factors to Ameliorate Cognitive Aging: A Systematic Review and Meta-analysis". figshare. https://hdl.handle.net/2134/20937. 


\title{
Individually modifiable risk factors to ameliorate cognitive aging: a systematic review and meta-analysis
}

\author{
P. Lehert, P. Villaseca", E. Hogervorst ${ }^{\dagger}, P . M$. Maki and V. W. Henderson ${ }^{* *}$
}

Faculty of Economics, Université Catholique de Louvain (UCL Mons), Mons, Belgium; "Department of Endocrinology, Faculty of Medicine, Pontificia Universidad Católica de Chile, Santiago, Chile; ${ }^{\dagger}$ Applied Cognitive Research, School of Sport, Exercise and Health Sciences, Loughborough University, Loughborough, Leicestershire, UK; ${ }^{\ddagger}$ Departments of Psychiatry and Psychology, University of Illinois at Chicago, Chicago, Illinois; USA; "Departments of Health Research and Policy (Epidemiology) and of Neurology and Neurological Sciences, Stanford University, Stanford, California, USA

Key words: COGNITION, COGNITIVE ACTIVITY, COGNITIVE AGING, DEHYDROEPIANDROSTERONE, FOLATE, GINKGO BILOBA, MENOPAUSAL HORMONE THERAPY, ISOFLAVONES, MEDITERRANEAN DIET, MEMORY, META-ANALYSIS, MINDFULNESS, OMEGA-3 FATTY ACIDS, PHYSICAL EXERCISE, SOCIAL ENGAGEMENT, SYSTEMATIC REVIEW,TAI CHI, VITAMIN BI2, VITAMIN D

\section{ABSTRACT}

A number of health and lifestyle factors are thought to contribute to cognitive decline associated with age but cannot be easily modified by the individual patient. We identified 12 individually modifiable interventions that can be implemented during midlife or later with the potential to ameliorate cognitive aging. For ten of these, we used PubMed databases for a systematic review of long-duration (at least 6 months), randomized, controlled trials in midlife and older adults without dementia or mild cognitive impairment with objective measures of neuropsychological performance. Using network meta-analysis, we performed a quantitative synthesis for global cognition (primary/outcome) and episodic memory (secondary outcome). Of 1038 publications identified by our search strategy, 24 eligible trials were included in the network meta-analysis. Results suggested that the Mediterranean diet supplemented by olive oil and tai chi exercise may improve global cognition, and the Mediterranean diet plus olive oil and soy isoflavone supplements may improve memory. Effect sizes were no more than small (standardized mean differences 0.11-0.22). Cognitive training may have cognitive benefit as well. Most individually modifiable risk factors have not yet been adequately studied. We conclude that some interventions that can be self-initiated by healthy midlife and older adults may ameliorate cognitiye aging.

\section{INTRODUCTION}

We do not focus directly on factors linked to the risk of dementia. Interventions that might prevent cognitive aging are not necessarily identical to those that might reduce risk of Alzheimer's disease or another dementia. There are, however, shared risk factors. Moreover, an intervention that ameliorates cognitive aging would be expected at the same time to reduce the likelihood of dementia by augmenting cognitive reserve, improving brain health, or both ${ }^{1}$. Cognitive reserve is enhanced by increasing the capacity, efficiency or redundancy of brain areas and neural pathways used when a cognitive task is performed ${ }^{2}$. Educational attainment, for example, is associated with reduced risk of dementia ${ }^{3}$. Brain health might be boosted by improved microcirculation, reduced oxidative
Each of us is responsible for our own health, and many aspect of healthy aging are under our direct control. With good reason, we are admonished to stop smoking, exercise regularty, and use sun screen. Concerns with memory and cognitive abilities are increasingly common in midlife and older adulthood. For cognitive aging, advice abounds, but it is less certain what the individual can do to maintain or improve mental abilities. The purpose of this systematic review is to evaluate evidence on (a) common, modifiable risk factors for (b) cognitive aging that are (c) largely under the individual's personal control and (d) can be implemented in midlife or later. 
stress, enhanced glymphatic clearance of toxic metabolites, and other mechanisms.

\section{Cognitive aging, mild cognitive impairment, and dementia}

Cognitive abilities change over the life span, and performances on many - but not all - cognitive tasks show decline during midlife and older adulthood. The most severe form of cognitive deterioration is dementia, also referred to as major neurocognitive impairment. Dementia is caused by specific brain pathologies, such as the neuritic plaques and neurofibrillary tangles of Alzheimer's disease or cerebral infarction characteristic of dementia due to cerebral vascular disease. In most instances, dementia is preceded by a stage of milder decline (mild cognitive impairment, or MCI $)^{4}$, where the overall pathological burden is less severe than in dementia.

Cognitive aging represents decline in the absence of specific dementia pathologies. The underlying physiological processes are poorly characterized but are not thought to eventuate in dementia, absent co-existing dementia pathologies. Cognitive aging and MCI, however, are not always easily distinguished, and by the tenth decade of life some degree of dementia pathology is near-universal.

\section{Midlife and beyond}

Our analyses focus on interventions that can be implemented in midlife or later, a time when cognitive concerns are heightened, and presumably before there is evidence of pathological decline indicative of MCI or dementia. We include men as well as women because - apart from hormonal exposures - many modifiable risk factors pertain to both sexes, and many clinical trials still do not report separate outcomes for women and men.

For women, midlife is conceptualized to begin with the menopausal transition, as the reproductive phase of a woman's life draws to a close. Natural menopause, defined retrospectively after 12 months of amenorrhea ${ }^{5}$, occurs at a median age of 51 years, and menstrual cycle irregularity characteristic of the menopausal transition begins on average about 4 years before. For men, where reductions in gonadal testosterone occur gradually throughout adult life ${ }^{6}$, midlife might somewhat arbitrarily be said to begin at age 50. For women and men, midlife continues up until age 65 years, the traditional threshold for older adulthood.

\section{Individually modifiable risk factors}

In their exhaustive report on preventing Alzheimer's disease, $\mathrm{MCI}$, and cognitive decline, Williams and colleagues ${ }^{7}$ tackled a broad range of exposures and interventions. A number of factors identified in their analyses are of public health import yet do not offer meaningful opportunities for at-risk individuals at midlife or older.

This dilemma is especially true for medical conditions. Important disorders considered by Williams and colleagues, such as diabetes mellitus, hypertension, hyperlipidemia, and depression, require treatment regardless of how the illness might - or might not - impact cognitive aging. For most prescription drugs, options for individual patients are similarly limited. Side-effect profiles and personal preference can help guide selection, but the decision whether or not to treat is usually not open to debate. Cigarette smoking can be viewed analogously. This lifestyle factor is strongly associated with cardiovascular disease, stroke, lung cancer, and overall mortality. Public health exhortations to stop smoking will be largely unaffected by cognitive considerations. The individual smoker already knows she should stop.

Williams and colleagues ${ }^{7}$ also discuss social factors associated with cognitive health. Some, however, cannot be addressed by middle-age and older adults. One's early childhood environment is not modifiable in adulthood. Most critical decisions on education or occupation are made well before midlife. Marital status can change at any age but would seem difficult to modify on the basis of cognitive concerns.

The midlife or older adult, however, has direct control over many lifestyle practices and nutritional factors. In addition, menopausal hormone therapy (MHT) is a notable exception to the non-discretionary nature of prescription drugs. For its most common indication - the treatment of moderate to severe vasomotor symptoms - its use is often viewed as discretionary. There are alternative forms of pharmacologic and nonpharmacologic therapies ${ }^{8}$, which are often recommended in preference to MHT. A woman's informed decision is increasingly the critical factor in whether MHT is prescribed.

\section{Risk factor selection}

58

Based on these considerations, we identified 12 individually modifiable factors. For ten of these, we undertook a systematic review and quantitative synthesis. For two others, we relied on recently published meta-analyses (Table 1). Each selected intervention can be implemented during or after midlife. For each, the key question was, 'What are the cognitive effects of the intervention?'. Because randomized, controlled trials provide the strongest evidence for causality, our systematic reviews and synthesis were based on clinical trial findings. We used other evidence, including findings from longitudinal observation and prior systematic reviews, to frame the issues and discuss our results.

\section{METHODS}

\section{Approach}

Our approach is given below and summarized in Table 2 . 
Table 1 Personally modifiable, midlife and older life interventions with the potential to ameliorate cognitive aging

\begin{tabular}{|c|c|}
\hline Factor & Classification \\
\hline B-vitamin supplements* & Nutritional supplement \\
\hline Dehydroepiandrosterone & $\begin{array}{l}\text { Nutritional supplement or } \\
\text { prescription } \text { drug }^{\dagger}\end{array}$ \\
\hline Ginkgo biloba extract & Nutritional supplement \\
\hline Mediterranean diet & Dietary factors \\
\hline Menopausal hormone therapy ${ }^{* *}$ & Prescription drug \\
\hline Mindfulness & Lifestyle \\
\hline $\begin{array}{l}\text { Omega-3 polyunsaturated fatty } \\
\text { acids }\end{array}$ & $\begin{array}{l}\text { Dietary factor or nutritional } \\
\text { supplement }\end{array}$ \\
\hline Social engagement & Lifestyle \\
\hline Soy isoflavones ${ }^{\dagger \dagger}$ & $\begin{array}{l}\text { Dietary factor or nutritional } \\
\text { supplement }\end{array}$ \\
\hline Vitamin D supplements ${ }^{\ddagger}$ & Nutritional supplement \\
\hline $\begin{array}{l}\text { Cognitive activity and cognitive } \\
\text { training }\end{array}$ & Lifestyle \\
\hline Physical activity (aerobic exercise) & Lifestyle \\
\hline
\end{tabular}

*, Folic acid, vitamin B12, and/or vitamin B6, not part of a broadly construed nutritional or multivitamin supplement; ${ }^{\dagger}$, dietary supplement in the US, controlled drug in most other countries; ${ }^{\ddagger}$, not part of a broadly construed nutritional or multivitamin supplement; **, oral, transdermal or parenteral, excludes topical (vaginal) formulations; ${ }^{\dagger \dagger}$, soy food products or soy isoflavone supplements

\section{Evidence}

Systematic searches were based on randomized, controlled trials involving a single active intervention and a placebo or presumably inactive comparator. Where blinding was feasiblefor example, when the intervention was a prescription drug or nutritional supplement - we sought confirmation that participants and evaluators were blinded. Where participant blinding was not feasible - for example, tai chi exercise or the Mediterranean diet - we required blinded outcome assessment. To reduce publication bias 9 , we required evaluable outcomes from at least 50 trial participants. Because we were interested in long-term, sustained cognitive benefit, we required at least 6 months between intervention initiation and outcome assessment.

\section{Participant characteristics}

Participants of eligible trials were midlife or older, recruited from a generally healthy population, and without MCI, dementia, or a specific medical disorder. We allowed at-risk populations (e.g. elevated serum concentrations of homocysteine) without end-organ disease (e.g. stroke). For samples with younger adults, the mean age had to be at least 50 years. We considered studies of women, men, and both sexes combined. Most trials included men and women; very few provided sex-specific cognitive outcome data that would allow an examination of possible interactions by sex. For hormonal interventions, we were interested in the possibility that a
Table 2 Inclusion and exclusion criteria for systematic review 58

\begin{tabular}{|c|c|}
\hline Category & Criteria \\
\hline Study populations & $\begin{array}{l}\text { Midlife and older adult women or men; } \\
\text { drawn from a generally healthy general } \\
\text { population; without dementia or mild } \\
\text { cognitive impairment }\end{array}$ \\
\hline Sample size & $\begin{array}{l}\text { At least } 50 \text { participants with evaluable } \\
\text { outcomes }\end{array}$ \\
\hline Interventions & See text and Table 1 \\
\hline Duration & 6 months or longer \\
\hline Evaluable outcomes & $\begin{array}{l}\text { Change in cognition, based on objective, } \\
\text { quantitative neuropsychological tests" }\end{array}$ \\
\hline $\begin{array}{l}\text { Primary cognitive } \\
\text { outcome }\end{array}$ & $\begin{array}{l}\text { Global cognition: based on all available } \\
\text { neuropsychological tests, including tests } \\
\text { of episodic memory, general intelligence, } \\
\text { and screening cognition }\end{array}$ \\
\hline $\begin{array}{l}\text { Secondary cognitive } \\
\text { outcome }\end{array}$ & $\begin{array}{l}\text { Episodic memory: based on tests of verbal } \\
\text { or non-verbal learning and recall }\end{array}$ \\
\hline
\end{tabular}

*, Excluded tests of 'premorbid' intelligence, such as tests of vocabulary or the pronunciation of orthographically irregular words, and tasks primarily/conceptualized as non-cognitive, such as fingertapping; ${ }^{\dagger}$, examples are the Benton Visual Retention Test, California Verbal Learning Test, Hopkins Verbal Learning Test, and paired- 85 associates learning. General intelligence encompassed tests of working 86 memory, executive functioning, semantic memory, perceptual speed, 87 and visuoconstruction. Examples of screening cognitive tests (screening 88 cognition) are the Mini-Mental State examination and the Telephone 89 Interview of Cognitive Status 90

woman's age or temporal proximity to menopause might 92 modify effects of the intervention. Few trials provided these 93 data, however, and we were unable to address issues of timing 94 in a systematic manner. $\quad 95$

Search strategy and data abstraction 98

We searched PubMed databases through May 2015 to identify 99 eligible trials in any language, as long as an English-language 100 abstract was available. To identify other clinical trials, we 101 examined reference lists from acquired trials and recent 102 meta-analyses. Medical Subject Heading (MeSH) search terms 103 and keywords for searches are in Supplemental Table S1, 104 to be found online at http://informahealthcare.com/doi/abs/ 105 10.3109/13697137.2015.1078106.

Using prespecified inclusion and exclusion criteria, 107 titles and abstracts were examined for potential relevance. 108 Neuropsychological tests were categorized as tests of memory 109 or general intelligence or as screening cognitive tests (Table 2). 110 Memory tests were conceptualized as representing cognitive 111 functions mediated by the hippocampus and adjacent medial 112 temporal lobe areas, and general intelligence tests as repre- 113 senting functions mediated by neocortical association cortex. 114 
Screening cognitive tests were relative short instruments that incorporated both memory and general intelligence items. Data from published reports were summarized in evidence tables by one reviewer and verified by a second. Other studies were reviewed qualitatively.

\section{Data synthesis}

We focused on continuous measures of cognitive function. Categorical ratings based on cut scores are often arbitrary, of uncertain clinical relevance, and fail to take advantage of the full range of information contained within a continuous measure. Although categorical ratings such as transition to MCI are clinically meaningful, they typically involve assessment of both cognitive and non-cognitive processes. The transition also implicates specific pathological processes, such as those linked to Alzheimer's disease ${ }^{10}$. We were interested in cognitive decline independent of non-cognitive change and without implicit links to inferred pathologies. Our primary endpoint was global cognition derived from all neuropsychological test scores. Our secondary outcome was memory based both on verbally mediated tests of episodic memory and on tests less amenable to verbal encoding and retrieval strategies. We recognize that some interventions might have relatively isolated, domain-specific effects, or that some effects might be positive within one cognitive domain and neutral or negative in another. However, we were particularly concerned with the net benefit or harm of an intervention on overall cognitive functioning and, secondarily, on overall memory skills.

\section{Statistical methods}

We undertook a network meta-analysis to examine effects of individually modifiable risk factors on cognitive outcomes. This approach combines information from multiple trials that compare two or more interventions for a given disorder and provides indirect comparisons between interventions in different studies ${ }^{11,12}$. Neuropsychological tests were identified as providing memory (secondary outcome), general intelligence or screening cognitive test outcomes (see Table 2 for examples). Our primary outcome (global cognition) used results of all tests. Within each study, effect size variances were adjusted to account for multiple comparisons and endpoints. For each activecontrol intervention, we calculated standardized mean differences (effect sizes) and adjusted standardized errors. Effect sizes of at least 0.2 but less than 0.5 are usually described as 'small'. We report nominally significant ( two-tailed $p<0.05$ ) standardized mean differences $\geq 0.1$ as having potential clinical relevance, and describe these differences as very small $(0.1$ to $<0.2)$ or small $(0.2$ to $<0.5)$. Our initial approach used fixed-effect models, under the assumption that interventions would have comparable effects on cognitive outcomes in other populations of healthy midlife and older populations. We used a random-effects model in sensitivity analyses ${ }^{13}$. Statistical analyses were performed using $\mathrm{R}$ statistical packages (release 3.2.0) and the meta-library Netmeta ${ }^{14}$.

\section{RESULTS}

Of the 1038 publications identified by our search strategy (see Supplementary Table S1, to be found online at http://informa healthcare.com/doi/abs/10.3109/13697137.2015.1078106), 24 eligible clinical trials were included in the network meta-analysis, with 490 treatment arms for three groups of cognitive endpoints (memory, general intelligence, screening cognition).

A funnel plot of the treatment effect versus standardized error of the treatment effect showed a balanced distribution, as evidence for absence of publication bias. Results of fixed-effect models for memory, general intelligence and screening cognition did not indicate heterogeneity among studies (Cochran $\left.\mathrm{Q}: p=0.21-0.91, I^{2}=0.0-8.4 \%, \tau^{2}<0.001-0.0012\right)$; results were similar for global cognition (Cochran Q: $p=0.31$, $\left.I^{2}=4 \%, \tau^{2}=0.0004\right)$. Similar findings for memory, general intelligence and screening cognition justified a general pooling of the network (Kendall rank correlation coefficient $=0.91$; good internal consistency (Cronbach $\alpha=0.89$ ); $73 \%$ of variance explained by the first principal component in a principal components analysis). Results from random effects models were virtually identical to those of fixed effect models (see Supplementary Tables S2 and S3, to be found online at http:// informahealthcare.com/doi/abs/10.3109/13697137.2015. 1078106). Some findings for the two Mediterranean diets and two mindfulness interventions (tai chi and yoga) differed significantly from each other and are described separately.

Most interventions had no significant effect on any cognitive outcome (results for global cognition and memory are shown in Figure 1). Two had significant positive effects on global cognition that were small (Mediterranean diet + olive oil: standardized mean difference $0.22,95 \%$ CI $0.16-0.27$ ) or very small (tai chi exercise: standardized mean difference 0.18 , 95\% CI 0.06-0.29). Two interventions had small (Mediterranean diet + olive oil: standardized mean difference 0.22 , 95\% CI 0.12-0.32) or very small (soy isoflavone supplements: standardized mean difference $0.11,95 \%$ CI $0.04-0.17)$ positive effects on memory. Nominally significant differences for global cognition below our threshold for potential clinical relevance were noted for MHT (negative: standardized mean difference $-0.03,95 \%$ CI -0.05 to -0.01 ), soy isoflavones (positive: standardized mean difference $0.04,95 \%$ CI $0.002-0.08$ ) and the Mediterranean diet + nuts (positive: standardized mean difference $0.08,95 \%$ CI $0.03-014)$.

\section{DISCUSSION}

\section{B-vitamins}

There is an intriguing relation between homocysteine, B-vitamins, and cognitive impairment. Homocysteine is a sulfur-containing 
Intervention

Global cognition

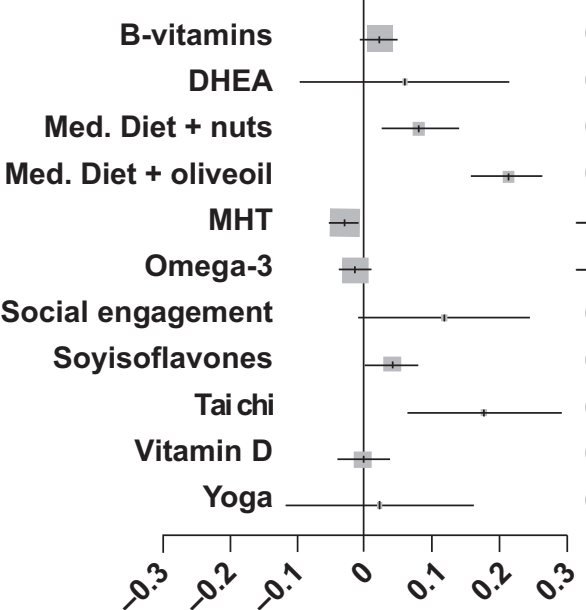

$0.06(-0.09,0.22)$

$0.08(0.03,0.14)$

$0.22(0.16, \quad 0.27)$

$-0.03(-0.05,-0.01)$

$-0.01(-0.04,0.01)$

$0.12(-0.01,0.25)$

$0.04(0.00,0.08)$

$0.18(0.06, \quad 0.29)$

$0.00(-0.04,0.04)$

$0.02(-0.12,0.16)$
$0.02(0.00, \quad 0.05)$
Episodic memory

$0.04(0.00, \quad 0.08)$

$0.07(-0.03,0.17)$

$0.22(0.12, \quad 0.32)$

$-0.01(-0.04,0.03)$

$0.02(-0.04,0.07)$

$0.20(-0.08,0.47)$

$0.11(0.04,0.17)$

$0.24(-0.03,0.51)$

$-0.01(-0.08,0.06)$

$0.06(-0.38,0.50)$
$0.12(-0.15,0.39)$

58

59

60

61

62

63

64

65

66

67

68

69

Figure 1 Results of the network meta-analysis. Fixed-effects model. Weighted standardized mean differences and $95 \%$ confidence intervals 76 by intervention for primary (global cognition) and secondary (episodic memory) outcomes. DHEA, dehydroepiandrosterone; Med. Diet, 77 Mediterranean diet; MHT, menopausal hormone therapy; Omega-3, omega-3 fatty acids 78 amino acid derived from methionine. Circulating levels increase with age, and higher homocysteine levels are associated with several important disorders, including coronary heart disease and Alzheimer's disease. Vitamin B12 (cobalamin), folic acid (vitamin B9), and vitamin B6 (pyridoxine) are cofactors in the conversion of methionine to homocysteine. Lower blood levels of folic acid and vitamin B12 are associated with Alzheimer's disease ${ }^{15}$, and B-vitamin supplements reduce homocysteine levels ${ }^{16}$.

Despite some encouraging findings - for example, less brain atrophy in MCI patients treated with folate and vitamin B12 17 - cognitive endpoints in randomized trials have often been null, both for dementia patients and for adults with normal cognition ${ }^{18}$.

Four clinical trials met our search criteria, conducted over periods of 2 or 3 years ${ }^{19-22}$. Each was limited to older adults; participants in three trials were preselected on the basis of elevated plasma homocysteine. The active interventions were folate $(400-2000 \mu \mathrm{g}$; four trials) plus vitamin B12 (400 or $500 \mu \mathrm{g}$; three trials) and vitamin B6 (10 or $25 \mathrm{mg}$; two trials). The B-vitamin interventions effectively lowered homocysteine levels. One trial reported improved memory and other cognitive skills with folate supplements ${ }^{19}$, and three reported no cognitive effect of B-vitamin intervention $^{20-22}$. Our meta-analysis indicated no benefit for global cognition or memory.

\section{Dehydroepiandrosterone}

Dehydroepiandrosterone (DHEA) is a weakly androgenic steroid secreted by the adrenal cortex. Small quantities are also produced within the brain. It is an intermediary in the biosynthesis of androgens and estrogens. DHEA or its sulfate ester has been hyped as a superhormone and as an anti-aging hormone. It is the most abundant circulating steroid, and levels in women and men decline dramatically with age. Interest in DHEA is particularly keen in the US, where it is classified as a dietary supplement and can be purchased over the counter. In most countries, it is available only by prescription, including the UK where it is regulated as a Class C drug.

A Cochrane review concluded that evidence did not support a beneficial effect of DHEA supplementation on cognitive function of middle-age or older adults without dementia ${ }^{23}$. One clinical trial met our search criteria. In this 12 -month US study, 225 midlife and older men and women were randomized to DHEA $50 \mathrm{mg}$ daily or placebo $^{24}$. Consistent with the interpretation of study authors, we identified no cognitive benefit.

\section{Ginkgo biloba}

Ginkgo biloba is extracted from leaves of the Ginkgo biloba tree, described as a living fossil unrelated to other extant tree species. The extract is marketed as a dietary supplement, often with claims that it boosts memory. It has been tested in patients with MCI and dementia, as well as cognitive aging. Smaller trials found Ginkgo biloba extract promising in stabilizing or slowing decline in cognitively impaired patients with neuropsychiatric symptoms ${ }^{25}$. However, very large clinical trials in the US and France found no evidence that Ginkgo biloba reduced the incidence of dementia over a 5- or 6-year period $^{26,27}$.

Fewer studies have assessed the effects of Ginkgo biloba on cognitive aging. Cognitive decline was assessed as a secondary outcome in the Ginkgo Evaluation of Memory trial ${ }^{27}$. The study enrolled over 3000 community-dwelling adults aged 
72 years and older. The study cohort included patients with MCI as well as cognitively normal participants. When compared to placebo, Ginkgo biloba extract over 6 years did not reduce declines in memory or other cognitive functions ${ }^{28}$. One clinical trial would have otherwise met our eligibility criteria, except that data were not in a form that we could extract for quantitative analysis. This 42-month US study of 118 cognitively normal participants over 84 years of age found no significant difference in memory decline between participants allocated to Ginkgo biloba or placebo ${ }^{29}$.

\section{Mediterranean diet}

The Mediterranean diet holds promise as a palatable approach to the remediation of cognitive aging. There is no one specific Mediterranean diet. Rather, the diet reflects traditional patterns of food consumption in Greece, southern Italy, Spain, and Portugal. Characteristics include relatively large proportions of fish and relatively low proportions of meat; unsaturated fatty acids such as those found in olive oil; legumes, fruits, vegetables, and unprocessed cereal grains; moderate amounts of cheese, yogurt, and other dairy products; and moderate quantities of wine. Observational research suggests that higher adherence to a Mediterranean diet is associated with lower risks of MCI and Alzheimer's disease ${ }^{30}$. In the Nurses' Health Study, long-term adherence to a Mediterranean diet was associated with moderately better cognition but was unrelated to cognitive change ${ }^{31}$.

One clinical trial met our search criteria. This was a multisite study of over 1000 Spanish participants aged 55-80 years with diabetes or other cardiovascular risk factors. Participants were randomized to one of two versions of the Mediterranean diet (supplemented with extra virgin oliye oil (Mediterranean diet + olive oil) or mixed nuts (Mediterranean diet + nuts)) or to a low-fat diet control diet. At the Navarra study site, cognitive function was screened 6.5 years after randomization $^{32}$, with detailed testing on a subset of participants ${ }^{33}$. At the Barcelona site, neuropsychological tests were administered at baseline and about 4 years later ${ }^{34}$. Substantial numbers of participants were lost to follow-up or excluded. Compared to the low-fat diet, both Mediterranean diets were reported to improve aspects of cognitive function. Our meta-analysis suggested better global cognition and memory with the Mediterranean diet + olive oil.

\section{Menopausal hormone therapy}

After menopause, the depletion of ovarian follicles leads to permanent reductions in circulating levels of estrogens and progesterone, although small amounts continue to be made within the brain. These hormonal changes can affect neural processes concerned with cognition and pathological processes linked to Alzheimer's disease.

Cognitive complaints are common during midlife, and the menopausal transition may represent a time of cognitive vulnerability ${ }^{35}$. It is controversial whether MHT, a systemic estrogen with or without a progestational agent, benefits or harms cognitive abilities ${ }^{36}$. A related controversy concerns MHT effects on Alzheimer's disease. Clinical trial evidence from the Women's Health Initiative (WHI) indicates that MHT increases dementia risk in women after age 65 years and older, whereas observational data link MHT use at younger ages to reduced Alzheimer risk ${ }^{37-39}$.

We identified six eligible trials for review and quantitative synthesis. All involved women aged 60 years and older. The MHT formulation in most trials was conjugated estrogens $0.625 \mathrm{mg} /$ day with or without medroxyprogesterone acetate ${ }^{40-45}$; other formulations were low-dose transdermal estradiol $0.014 \mathrm{mg} / \mathrm{day}^{46}$ and oral estradiol $1 \mathrm{mg} / \mathrm{day}$ and norethindrone ${ }^{47}$. Most comparisons with placebo were nil. In single studies, differences favored placebo on a screening cognitive test $\mathrm{t}^{40}$ and a test of verbal memory ${ }^{42}$ and favored MHT on a non-verbal memory test ${ }^{42}$. Our meta-analysis of the six trials suggested no clinically meaningful effect of MHT compared to placebo on gløbal cognition or memory, with nominal effects on global cognition (standardized mean difference -0.03) that favored placebo.

Few clinical trials of MHT have included younger postmenopausal women, and none met our inclusion criteria. Small clinical trials in surgically menopausal women suggest short-term cognitive benefit of MHT when started at the time of oophorectomy ${ }^{36}$. A large 4-month trial of recently menopausal women with cognitive complaints found no cognitive benefit of conjugated estrogens $0.625 \mathrm{mg} /$ day plus medroxyprogesterone acetate in women aged $45-55$ years ${ }^{48}$. A threearm trial published too late to be included in our systematic review provides results from 693 younger postmenopausal women, mean age 53 years. Women were allocated to conjugated estrogens $0.45 \mathrm{mg} /$ day and oral micronized progesterone, to transdermal estradiol $0.05 \mathrm{mg} /$ day and micronized progesterone, or to placebo ${ }^{49}$. Cognitive outcomes at nearly 3 years did not differ significantly among treatment groups. The timing hypothesis is examined more directly in a large, recently completed randomized trial that includes both younger and older postmenopausal women randomized to oral estradiol with or without micronized progesterone, vaginal gel or placebo ${ }^{50}$; these findings are not yet published.

\section{Mindfulness}

Mindfulness is a mental state characterized by the focus of attention on the present moment. The attentional spotlight often includes bodily sensations - for example, proprioceptive sensations related to breathing or posture - as well as stimuli arising from the external environment. Mindfulness is intentional, non-analytical, and non-judgmental. It is an important component of meditation and mind-body practices such as yoga, tai chi, and qi gong. Mindfulness interventions have been most studied in relation to psychological stress, anxiety, and depression. Some investigators have examined cognitive outcomes as well. A recent meta-analysis of tai chi 
trials concluded that tai chi improves executive cognitive functions ${ }^{51}$.

Our systematic search focused on meditation, yoga, tai chi, and qi gong. We identified three eligible trials. A 6-month trial of Hatha yoga found no cognitive benefit compared to wait-list controls ${ }^{52}$. In contrast, tai chi exercises performed for 6 months (US) ${ }^{53}$ or 40 weeks (Shanghai, China) ${ }^{54}$ improved several neuropsychological measures. The Shanghai investigators reported significant increases in brain volume in the tai chi group compared to the no-intervention control ${ }^{54}$. Our meta-analysis indicates that tai chi exercise improves global cognition.

\section{Omega-3 polyunsaturated fatty acids}

Low rates of cardiovascular disease among the Inuit of Greenland are associated with high dietary intakes of fish. This observation led to studies on health effects of omega-3 fatty acids. These are n-3 long-chain polyunsaturated fatty acids, where n-3 refers to the location of the last carboncarbon double bond, three carbons from the end of the fatty acid backbone. Two, docosahexaenoic acid (DHA) and eicosapentaenoic acid (EPA), are obtained primarily from certain fatty fish and their oils. The brain contains large amounts of DHA, an important component of nerve cell membranes. A Cochrane review found no clear role for omega-3 fatty acids in modifying dementia risk and no clear benefit of omega-3 supplementation on cognitive abilities in healthy older adults ${ }^{55}$.

Three clinical trials met our search criteria. Active interventions were capsule supplements of EPA-DHA or ethyl-esters of n-3 polyunsaturated fatty acids ${ }^{56-58}$. The largest - a multinational trial targeting midlife and older adults with mild diabetes, abnormal fasting glucose levels, or impaired glucose tolerance - followed several thousand participants for a median of 6.2 years $^{56}$. None of the studies reported cognitive benefit compared to placebo, and our meta-analysis confirmed the absence of cognitive effect.

\section{Social engagement}

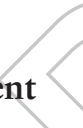

Social engagement is postulated to reduce risk for cognitive aging and dementia. Social engagement has been variously assessed - usually by self-report - from marital status, number of people within a household, size of social network, or participation in social activities. Observational findings on social engagement and cognition are inconsistent ${ }^{7}$.

Clinical trials that assess social engagement typically use a design that introduces other activities at the same time. For example, a pilot trial of volunteer service in elementary school settings provided participants with not only new social networks but also with new cognitive challenges and enhanced physical activity ${ }^{59}$. This multimodal approach is quite reasonable but makes it difficult to discern the contribution of social engagement per se.
One trial met our search criteria, the 40-week clinical 58 trial conducted in Shanghai, China, referred to above, which 59 included a social interaction arm and a no-intervention con- 60 trol $^{54}$. Social interaction occurred within an 'extremely lively' 61 discussion group that met for 1 hour, three times weekly under 62 the direction of a group leader. We did not find a significant 63 effect of social engagement on cognitive outcomes. 64

\section{Soy isoflavones}

Isoflavones are plant-derived diphenolic compounds structur- 69 ally similar to estrogens. They are sometimes classified as 70 selective estrogen receptor modulators, since biological effects 71 can be estrogenic or antiestrogenic in the brain and in other 72 tissues. Soy, the major dietary source of isoflavones, is a staple 73 of traditional diets in some Asian countries. Soy isoflavones 74 have been investigated in relation to breast cancer, prostate 75 cancer, cardiovascular disease, menopausal vasomotor symp- 76 toms, osteoporosis, and other health outcomes. Observational 77 studies in countries where soy dietary consumption is rela- 78 tively low generally report no associations with cognition. 79 Some investigations in populations with higher levels of 80 consumption report adyerse associations ${ }^{60,61}$. Cognitive effects 81 of different soy) products might differ ${ }^{61}$.

Four clinical trials, all involving healthy postmenopausal 83 women, met our search criteria: two from the US ${ }^{62,63}$, one 84 from Hong Kong ${ }^{64}$, and one from the Netherlands ${ }^{65}$. Sample 85 sizes ranged from 53 to 313, with follow-up times of 6 to 86 30 months. The active interventions were $80-110 \mathrm{mg}$ daily 87 of soy-derived isoflavone supplements. Where specified, 88 supplements contained genistein, daidzein, and glycitein in 89 the approximate ratio found in soy.

Most comparisons between treatment groups did not differ. 91 One trial reported better category fluency in the isoflavone 92 group $^{62}$ and one trial reported worse performance on a work- 93 ing memory task and better performance on a visual memory 94 task $^{63}$. The largest, longest trial found no treatment effect on 95 a composite neuropsychological measure of global cognition 96 but better performance in the isoflavone group on a composite 97 measure of visual memory ${ }^{63}$. In this trial, treatment group 98 comparisons on composite neuropsychological measures did 99 not differ between women less than age 60 years compared to 100 women aged 60 and above. In secondary analyses, there was 101 an inverse association between the level of endogenous expo- 102 sure (measured by urinary isoflavonoids) and performance 103 on neuropsychological tests of general intelligence (but not 104 memory $)^{66}$. Our meta-analysis indicated that soy isoflavone 105 supplements improve memory but have no effect on global 106 $\begin{array}{ll}\text { cognition. } & 107\end{array}$

\section{Vitamin D}

Vitamin D refers to several related fat-soluble steroid deriva- 112 tives, including vitamin D3 (1,25-dihydroxycholecalciferol, or 113 1,25-dihydroxyvitamin D) and vitamin D2 (ergocalciferol). 114 
Few foods contain vitamin D, and vitamin D deficiency is common in many areas of the world ${ }^{67}$. Dietary vitamin D3 is obtained from fish oils and fortified dairy products. The major natural source comes from conversion of 7-hydroxycholesterol to cholecalciferol in the skin in the presence of sunlight (ultraviolet B). Cholecalciferol is converted in the liver to 25 -hydroxyvitamin $\mathrm{D}$, which in turn is converted in the kidneys to vitamin D3, the biologically active form. Vitamin D3 crosses the blood-brain barrier and is locally synthesized in the brain from 25-hydroxyvitamin D. Cell-specific gene regulation occurs through interactions with the vitamin D receptor, a member of the steroid/thyroid hormone receptor superfamily. The receptor is widely distributed in the brain and other tissues.

Serum levels of 25-hydroxyvitamin D are lower in Alzheimer's disease patients than healthy older adults ${ }^{68}$, and lower levels are associated with poorer cognitive function and increased Alzheimer risk ${ }^{69}$. An Institute of Medicine report, however, found insufficient support for vitamin D benefit beyond recognized roles in calcium metabolism and bone health ${ }^{70}$.

One clinical trial met our search criteria ${ }^{71}$. This was a secondary analysis from the WHI trial of calcium and vitamin D (400 IU vitamin D3 daily) versus placebo. Many participants were simultaneously enrolled in the memory study component of the WHI MHT trial. Over a mean follow-up of more than 7 years, average scores on a screening cognitive test did not differ between treatment groups, nor did other neuropsychological test scores in a subgroup included in an ancillary analysis ${ }^{71}$. Our meta-analysis showed no eognitive effect of vitamin D.

\section{Cognitive and physical activities}

We did not undertake systematic reviews of cognitiye activity and physical activity because these lifestyle interventions have been widely publicized, and recent meta-analyses provide a basis for interpretation and conclusions.

\section{Cognitive activity and cognitive training}

Use-dependent neural plasticity forms the basis of learning, memory, and skill acquisition. Engaging in cognitively stimulating activity has the potential to ameliorate cognitive abilities diminished by aging. The Advanced Cognitive Training for Independent and Vital Elderly (ACTIVE) trial provides partial support for the mantra, 'use it or lose it'. This largescale randomized trial in community-dwelling older adults used interventions focused on memory, reasoning, or processing speed ${ }^{72}$. Comparisons were to a no-intervention control. Training occurred in group sessions over a period of about 5 weeks, and booster sessions were provided to a subset of participants. At 2 years, each active intervention improved cognitive skills within the targeted domain but not other cognitive domains ${ }^{72}$. Effects of the reasoning and processing speed interventions could still be detected 10 years later ${ }^{73}$. Training had no effect on everyday functioning at 2 years, but at 10 years self-reported functioning had declined less in cognitive training groups compared to the no-intervention $\operatorname{group}^{72,73}$.

A recent systematic review identified 31 randomized trials of cognitive training or mental stimulation involving older adults without known existing cognitive impairment ${ }^{74}$. Compared to no intervention, cognitive training significantly improved performance on several memory measures (facename recall, immediate recall, and paired associates learning, but not delayed recall). Compared to active controls, cognitive training improved performance on tasks involving memory (recognition) and other cognitive abilities (working memory, processing speed, and overall cognitive functioning). Similar findings were reported in a preceding metaanalysis $^{75}$.

Aerobic physical activity

$$
\text { exercise is thought to }
$$

Aerobic exercise is thought to maintain brain health indirectly through cardiovascular benefit and directly through effects on cerebral flow, neurogenesis, increased production of brain-derived neurotrophic factor, and other mechanisms. A robust animal literature supports a role for aerobic activity in maintaining cognitive function and reducing brain pathology in animal models of Alzheimer's disease. The observational literature extends these findings to humans, showing inverse associations between regular physical exercise and cognitive decline, MCI, and Alzheimer's disease $\mathrm{e}^{7,76,77}$. Aerobic exercise (walking) compared to stretching exercise is reported to increase the size of the anterior hippocampus $^{78}$.

A Cochrane Collaboration review assessed cognitive effects of aerobic exercise in 12 randomized trials ${ }^{79}$. Participants were aged 55 years and older. No intervention exceeded 6 months. There were three 6 -month trials, two with at least 50 participants ${ }^{52,80}$. The first trial randomly assigned sedentary, healthy older adults to an aerobic (walking) or anaerobic (toning and stretching) intervention, with structured classes that met three times weekly ${ }^{80}$. Executive control processes improved in the walking group. The second was a three-armed trial that included walking (one class weekly plus home exercise) and a wait-list control ${ }^{52}$. Cognitive function at trial completion did not differ between groups. In a 40-week trial not included in the Cochrane review, cognitive outcomes did not differ between participants in a thrice-weekly walking group and a no-intervention comparison group ${ }^{54}$. The Cochrane meta-analysis reported no evidence for cognitive benefit when aerobic exercise was compared to an active intervention (eight trials including 506 participants) or to no intervention at all (six trials, 296 participants $)^{79}$. Improved cardiorespiratory fitness was not associated with cognitive improvement. The authors concluded that aerobic exercise, including activities that 
improve cardiovascular fitness, provides no cognitive benefit in healthy older adults.

\section{General discussion}

A number of factors under an individual's control might improve cognitive aging and - although not a focus of this review - at the same time reduce dementia risk through enhanced cognitive reserve and brain health. Unfortunately, evidence in many areas is still inadequate. This is true not only for medical and psychiatric disorders, most prescription medications, and early life exposures ${ }^{7}$ but also for the individually modifiable factors considered in this review. Only four interventions in our meta-analysis included data from three or more clinical trials (B-vitamins, omega-3 polyunsaturated fatty acids, MHT, and soy isoflavones). Wide confidence intervals for some treatment effects (Figure 1) reflect the small number of trials and relatively small sample sizes.

Most interventions considered in our meta-analysis did not show clinically meaningful effects on global cognition or memory, and none showed effects that could be characterized as large, or even medium. Cognitive efficacy of the Mediterranean diet was supported by just one trial, with data from two study sites. It is difficult to know which components of this multifaceted nutritional intervention contributed to observed benefit. Because benefit in this dietary trial was most apparent in the arm receiving olive oil supplements, findings may not generalize to other versions of the Mediterranean diet.

Tai chi exercise also emerged as an intervention that may benefit cognitive aging. We identified only two eligible trials. We classified tai chi as a mindfulness intervention, but this Eastern exercise also involves skill learning and aerobic activity of mild-to-moderate intensity, taught in a socially engaging group setting. Beneficial effects of soy isoflavone supplements on memory (but not global cognition) and effects of cognitive training are other promising avenues for additional research. It should pointed out that isoflavone trials involved only women. High isoflavone dosages in these trials approximate levels of dietary consumption in several Asian countries but greatly exceed levels found in Western diets ${ }^{81}$. Our MHT results support guideline recommendations that MHT should not be used to ameliorate cognitive aging ${ }^{82,83}$. However, it

is important to recognize that few clinical trials of MHT 58 have included younger postmenopausal women and none has 59 focused specifically on the largest group of women for whom 60 MHT is indicated, namely women with moderate-to-severe 61 vasomotor symptoms.

There are limitations to our findings. We were unable to 63 consider all individually modifiable risk factors, and our 64 search strategy may not have identified all eligible trials for 65 factors that we did consider. An intervention might reduce 66 dementia risk without necessarily improving cognitive aging. 67 Exclusion of small trials to reduce publication bias could 68 introduce other biases, and we did not formally evaluate trial 69 quality. The focus on single interventions may underestimate 70 effects of multimodal or combined approaches. Cognitive 71 aging does not begin at midlife ${ }^{84}$, and effects of some individu- 72 ally modifiable interventions may be greater if implemented 73 at an earlier age. $\quad 74$

We conclude that individual choices can and do affect cog- 75 nitive aging. Beneficial effects, when present, are likely to be 76 modest but are nonetheless potentially important. However, 77 we do not make specific recommendations in the absence 78 of stronger evidence of meaningful effectiveness. Further 79 research, particularly on dietary factors, cognitive activity, 80 and multimodal leisure activities such as tai chi exercise seem 81 especially warranted.

\section{ACKNOWLEDGEMENTS 85}

86

We thank Rachael Smith and Barbara C. Henderson for 87 assistance with data verification.

Conflict of interest Philippe Lehert, Eef Hogervorst and Victor Henderson declare no conflict of interest for this study. Paulina Villaseca has received honoraria for lectures 93 and for acting as a member of an Advisory Board for Glaxo 94 Smith \& Kline. Pauline Maki has served as a consultant for 95 Noven, Abbott, and Pfizer.

Source of funding Supported by National Institutes of 99 Health grants R01AG034639 and P50AG047366. 100

\section{References}

1. Henderson VW. Three midlife strategies to prevent cognitive impairment due to Alzheimer's disease. Climacteric 2014; 17(Suppl 2):38-46

2. Stern Y. Cognitive reserve in aging and Alzheimer's disease. Lancet Neurol 2012;11:1006-12

3. Meng X, D'Arcy C. Education and dementia in the context of the cognitive reserve hypothesis: a systematic review with metaanalyses and qualitative analyses. PloS One 2012;7:e38268
4. Gauthier S, Reisberg B, Zaudig M, et al. Mild cognitive impairment. Lancet 2006;367:1262-70

5. Harlow SD, Gass M, Hall JE, et al. Executive summary of the Stages of Reproductive Aging Workshop + 10: addressing the unfinished agenda of staging reproductive aging. Climacteric 2012;15:105-14

6. Feldman HA, Longcope C, Derby CA, et al. Age trends 113 in the level of serum testosterone and other hormones in 114 
middle-aged men: longitudinal results from the Massachusetts Male Aging Study. J Clin Endocrinol Metab 2002;87: 589-98

7. Williams JW, Plassman BL, Burke J, Holsinger T, Benjamin S. Preventing Alzheimer's Disease and Cognitive Decline. Evidence Report/Technology Assessment Number 193. AHRQ Publication No. 10-E005. Rockville, MD: Department of Health and Human Services, 2010

8. Villaseca P. Non-estrogen conventional and phytochemical treatments for vasomotor symptoms: what needs to be known for practice. Climacteric 2012;15:115-24

9. Song F, Parekh S, Hooper L, et al. Dissemination and publication of research findings: an updated review of related biases. Health Technol Assess 2010;14:1-193

10. Albert MS, Dekosky ST, Dickson D, et al. The diagnosis of mild cognitive impairment due to Alzheimer's disease: Recommendations from the National Institute on Aging and Alzheimer's Association workgroup. Alzheimers Dement 2011;7:270-9

11. Rucker G. Network meta-analysis, electrical networks and graph theory. Res Synth Methods 2012;3:312-24

12. Krahn U, Binder H, Konig J. A graphical tool for locating inconsistency in network meta-analyses. BMC Med Res Methodol 2013;13:35

13. Lumley T. Network meta-analysis for indirect treatment comparisons. Stat Med 2002;21:2313-24

14. Rücker G, Schwarzer G, Krahn U, Jochem König J. Package 'netmeta', version 0.8-0, network meta-analysis using frequentist methods. R Library, Repository CRAN 2015-06-26 $18: 23: 34$

15. Shen L, Ji HF. Associations between homocysteine, folic acid, vitamin B12 and Alzheimer's disease: insights from meta-analyses. J Alzheimers Dis 2015 April 8. Epub ahead of print

16. Homocysteine Lowering Trialists' Collaboration. Lowering blood homocysteine with folic acid based supplements: meta-analysis of randomised trials. BMJ 1998;316:894-8

17. Douaud G, Refsum H, de Jager CA, et al. Preventing Alzheimer's disease-related gray matter atrophy by B-vitamin treatment. Proc Natl Acad Sci USA 2013;110:9523-8

18. Ford AH, Almeida OP. Effect of homocysteine lowering treatment on cognitive function: a systematic review and meta-analysis of randomized controlled trials. I Alzheimers Dis 2012;29: 133-49

19. Durga J, van Boxtel MP, Schouten EG, et al. Effect of 3-year folic acid supplementation on cognitive function in older adults in the FACIT trial: a randomised, double blind, controlled trial. Lancet 2007;369:208-16

20. McMahon JA, Green TJ, Skeaff CM, Knight RG, Mann JI, Williams SM. A controlled trial of homocysteine lowering and cognitive performance. N Engl J Med 2006;354:2764-72

21. van der Zwaluw NL, Dhonukshe-Rutten RA, van Wijngaarden JP, et al. Results of 2-year vitamin B treatment on cognitive performance: secondary data from an RCT. Neurology 2014;83: 2158-66

22. Ford AH, Flicker L, Alfonso $\mathrm{H}$, et al. Vitamins $\mathrm{B}(12), \mathrm{B}(6)$, and folic acid for cognition in older men. Neurology 2010; 75:1540-7

23. Grimley Evans J, Malouf R, Huppert F, van Niekerk JK. Dehydroepiandrosterone (DHEA) supplementation for cognitive function in healthy elderly people. Cochrane Database Syst Rev 2006:CD006221

24. Kritz-Silverstein D, von Muhlen D, Laughlin GA, Bettencourt R. Effects of dehydroepiandrosterone supplementation on cognitive function and quality of life: the DHEA and Well-Ness (DAWN) Trial. J Am Geriatr Soc 2008;56:1292-8
25. Tan MS, Yu JT, Tan CC, et al. Efficacy and adverse effects of Ginkgo biloba for cognitive impairment and dementia: a systematic review and meta-analysis. J Alzheimers Dis 2015;43: 589-603

26. Vellas B, Coley N, Ousset PJ, et al. Long-term use of standardised Ginkgo biloba extract for the prevention of Alzheimer's disease (GuidAge): a randomised placebo-controlled trial. Lancet Neurol 2012;11:851-9

27. DeKosky ST, Williamson JD, Fitzpatrick AL, et al. Ginkgo biloba for prevention of dementia: a randomized controlled trial. JAMA 2008;300:2253-62

28. Snitz BE, O'Meara ES, Carlson MC, et al. Ginkgo biloba for preventing cognitive decline in older adults: a randomized trial. JAMA 2009;302:2663-70

29. Dodge HH, Zitzelberger T, Oken BS, Howieson D, Kaye J. A randomized placebo-controlled trial of Ginkgo biloba for the prevention of cognitive decline. Neurology 2008;70:1809-17

30. Singh B, Parsaik AK, Mielke MM, et al. Association of Mediterranean diet with mild cognitive impairment and Alzheimer's disease: a systematic review and meta-analysis. J Alzheimers Dis 2014;39:271-82

31. Samieri C, Grodstein F, Rosner BA, et al. Mediterranean diet and cognitive function in older age. Epidemiology 2013;24:490-9

32. Martinez-Lapiscina EH, Clavero P, Toledo E, et al. Mediterranean diet improves cognition: the PREDIMED-NAVARRA randomised trial. V Neurol Neurosurg Psychiatry 2013;84: $1318-25$

33. Martinez-Lapiscina EH, Clavero P, Toledo E, et al. Virgin olive oil supplementation and long-term cognition: the PREDIMEDNAVARRA randomized trial. J Nutr Health Aging 2013;17: 544-52

34. Valls-Pedret C, Sala-Vila A, Serra-Mir M, et al. Mediterranean diet and age-related cognitive decline: a randomized clinical trial. JAMA Intern Med 2015;175:1094-103

35. Weber MT, Maki PM, McDermott MP. Cognition and mood in perimenopause: a systematic review and meta-analysis. J Steroid Biochem Mol Biol 2014;142:90-8

36. Hogervorst E, Bandelow S. Sex steroids to maintain cognitive function in women after the menopause: a meta-analysis of treatment trials. Maturitas 2010;66:56-71

37. Shumaker SA, Legault C, Kuller L, et al. Conjugated equine estrogens and incidence of probable dementia and mild cognitive impairment in postmenopausal women: Women's Health Initiative Memory Study. JAMA 2004;291:2947-58

38. Henderson VW, Espeland MA, Hogan PE, et al. Prior use of hormone therapy and incident Alzheimer's disease in the Women's Health Initiative Memory Study [abstract]. Neurology 2007; 68(Suppl 1):A205

39. Henderson VW. Alzheimer's disease: Review of hormone therapy trials and implications for treatment and prevention after menopause. J Steroid Biochem Mol Biol 2014;142:99-106

40. Rapp SR, Espeland MA, Shumaker SA, et al. The effect of estrogen with progestin treatment on global cognitive function in postmenopausal women: results from the Women's Health Initiative Memory Study. JAMA 2003;289:2663-72

41. Espeland MA, Rapp SR, Shumaker SA, et al. Conjugated equine estrogens and global cognitive function in postmenopausal women: Women's Health Initiative Memory Study. JAMA 2004;291:2959-68

42. Resnick SM, Maki PM, Rapp SR, et al. Effects of combination estrogen plus progestin hormone treatment on cognition and affect. J Clin Endocrinol Metab 2006;91:1802-10

43. Resnick SM, Espeland MA, An Y, et al. Effects of conjugated equine estrogens on cognition and affect in postmenopausal 
women with prior hysterectomy. J Clin Endocrinol Metab 2009; 94:4152-61

44. Binder EF, Schechtman KB, Birge SJ, Williams DB, Kohrt WM. Effects of hormone replacement therapy on cognitive performance in elderly women. Maturitas 2001;38:137-46

45. Greenspan SL, Resnick NM, Parker RA. The effect of hormone replacement on physical performance in community-dwelling elderly women. Am J Med 2005;118:1232-9

46. Yaffe K, Vittinghoff E, Ensrud KE, et al. Effects of ultra-low-dose transdermal estradiol on cognition and health-related quality of life. Arch Neurol 2006;63:945-50

47. Tierney MC, Oh P, Moineddin R, et al. A randomized doubleblind trial of the effects of hormone therapy on delayed verbal recall in older women. Psychoneuroendocrinology 2009;37: 1065-74

48. Maki PM, Gast MJ, Vieweg A, Burriss SW, Yaffe K. Hormone therapy in menopausal women with cognitive complaints: a randomized, double-blind trial. Neurology 2007;69:1322-30

49. Gleason CE, Dowling NM, Wharton W, et al. Effects of hormone therapy on cognition and mood in recently postmenopausal women: findings from the randomized, controlled KEEPS-cognitive and affective study. PLoS Med 2015;12:e1001833

50. Henderson VW, St. John JA, Hodis HN, et al. Cognition, mood, and physiological concentrations of sex hormones in the early and late postmenopause. Proc Natl Acad Sci USA 2013;110: 20290-5

51. Wayne PM, Walsh JN, Taylor-Piliae RE, et al. Effect of Tai Chi on cognitive performance in older adults: systematic review and meta-analysis. J Am Geriatr Soc 2014;62:25-39

52. Oken BS, Zajdel D, Kishiyama S, et al. Randomized, controlled, six-month trial of yoga in healthy seniors: effects on cognition and quality of life. Altern Ther Health Med 2006;12:40-7

53. Taylor-Piliae RE, Newell KA, Cherin R, Lee M, King AC, Haskell WL. Tai Chi versus Western exercise on physical and cognitive functioning in healthy community-dwelling older adults: a randomized clinical trial. J Aging Phys Act 2010;18:261-79

54. Mortimer JA, Ding D, Borenstein AR, et al. Changes in brain volume and cognition in a randomized trial of exercise and social interaction in a community-based sample of non-demented Chinese elders. J Alzheimers Dis 2012;30:757-66

55. Sydenham E, Dangour AD, Lim WS. Omega 3 fatty acid for the prevention of cognitive decline and dementia. Cochrane Database Syst Rev 2012:CD005379

56. Cukierman-Yaffe T, Bosch J, Diaz R, et al. Effects of basal insulin glargine and omega- 3 fatty acid on cognitive decline and probable cognitive impairment in people with dysglycaemia: a substudy of the ORIGIN trial. Lancet Diabetes Endocrinol 2014;2:562-72

57. van de Rest O, Geleijnse JM, Kok FJ, et al. Effect of fish oil on cognitive performance in older subjects: a randomized, controlled trial. Neurology 2008;71:430-8

58. Dangour AD, Allen E, Elbourne D, et al. Effect of 2-y n-3 longchain polyunsaturated fatty acid supplementation on cognitive function in older people: a randomized, double-blind, controlled trial. Am J Clin Nutr 2010;91:1725-32

59. Carlson MC, Saczynski JS, Rebok GW, et al. Exploring the effects of an "everyday" activity program on executive function and memory in older adults: Experience Corps. Gerontologist 2008;48:793-801

60. White LR, Petrovitch H, Ross GW, et al. Brain aging and midlife tofu consumption. J Am Coll Nutr 2000;19:242-55

61. Soni M, Rahardjo TB, Soekardi R, et al. Phytoestrogens and cognitive function: a review. Maturitas 2014;77:209-20

62. Kritz-Silverstein D, Von Muehlen D, Barrett-Connor E, Bressel MAB. Isoflavones and cognitive function in older women: the SOy and Postmenopausal Health in Aging (SOPHIA) 58 Study. Menopause 2003;10:196-202

63. Henderson VW, St John JA, Hodis HN, et al. Long-term soy isoflavone supplementation and cognition in women: A rand- 61 omized, controlled trial. Neurology 2012;78:1841-8

64. Ho SC, Chan AS, Ho YP, et al. Effects of soy isoflavone supplementation on cognitive function in Chinese postmenopausal women: a double-blind, randomized, controlled trial. Menopause 2007;14:489-99

65. Kreijkamp-Kaspers S, Kok L, Grobbee DE, et al. Effect of soy 66 protein containing isoflavones on cognitive function, bone min- 67 eral density, and plasma lipids in postmenopausal women: a randomized controlled trial. JAMA 2004;292:65-74

66. St John JA, Henderson VW, Hodis HN, et al. Associations between urine excretion of isoflavonoids and cognition in postmenopausal women in the Women's Isoflavone Soy Health clinical trial. J Am Geriatr Soc 2014;62:629-35

67. Holick MF. Vitamin D deficiency. N Engl J Med 2007;357: 266-81 toy S, Duque G, Beauchet O. Meta-analysis of memory and executive dysfunctions in relation to yitamin D. J Alzheimers Dis 2013;37:147-71

69. Balion C, Griffith LE, Strifler D, et al. Vitamin D, cognition, and 78 dementia: a systematic review and meta-analysis. Neurology 79 2012;79:1397-405

70. Ross AC, Taylor CL, Yaktine AL, Del Valle HB, eds. Dietary Reference Intakes for Calcium and Vitamin D. Washington, DC: National Academies Press, 2011

71. Rossom Re, Espeland MA, Manson JE, et al. Calcium and vitamin D supplementation and cognitive impairment in the Women's Health Initiative. J Am Geriatr Soc 2012;60:2197-205

72. Ball K, Berch DB, Helmers KF, et al. Effects of cognitive training 86 interventions with older adults: a randomized controlled trial. JAMA 2002;288:2271-81

73. Rebok GW, Ball K, Guey LT, et al. Ten-year effects of the advanced cognitive training for independent and vital elderly cognitive training trial on cognition and everyday functioning in older adults. J Am Geriatr Soc 2014;62:16-24

74. Kelly ME, Loughrey D, Lawlor BA, Robertson IH, Walsh C, 92 Brennan S. The impact of cognitive training and mental stimula- 93 tion on cognitive and everyday functioning of healthy older 94 adults: a systematic review and meta-analysis. Ageing Res Rev 95 2014; 15:28-43

75. Reijnders J, van Heugten C, van Boxtel M. Cognitive interventions in healthy older adults and people with mild cognitive impairment: a systematic review. Ageing Res Rev 2013;12:26375

76. Sofi F, Valecchi D, Bacci D, et al. Physical activity and risk of cognitive decline: a meta-analysis of prospective studies. J Intern Med 2011;269:107-17

77. Geda YE, Roberts RO, Knopman DS, et al. Physical exercise, aging, and mild cognitive impairment: a population-based study. Arch Neurol 2010;67:80-6

78. Erickson KI, Voss MW, Prakash RS, et al. Exercise training increases size of hippocampus and improves memory. Proc Natl Acad Sci USA 2011;108:3017-22

79. Young J, Angevaren M, Rusted J, Tabet N. Aerobic exercise to improve cognitive function in older people without known cognitive impairment. Cochrane Database Syst Rev 2015;4: CD005381

80. Kramer AF, Hahn S, McAuley E, et al. Exercise, aging and 111 cognition: healthy body, healthy mind? In Fish AD, Rogers W, 112 eds. Human Factors Interventions for the Health Care of Older 113 Adults. Hillsdale, NJ: Erlbaum, 2001:91-120 78 78
79 87 88 89 90 91 9
9 96 97 98 2 
81. Messina M, Nagata C, Wu AH. Estimated Asian adult soy protein and isoflavone intakes. Nutr Cancer 2006;55:1-12

82. de Villiers TJ, Pines A, Panay N, et al. Updated 2013 International Menopause Society recommendations on menopausal hormone therapy and preventive strategies for midlife health. Climacteric 2013;16:316-37

\section{Supplementary materials available online}

Table S1 Search terms used in PubMed searches

Table S2 Cognitive effects of individually modifiable factors, random-effects model

Table S3 Effects of individually modifiable interventions: general intelligence (non-memory) outcomes
83. Shifren JL, Gass ML, NAMS Recommendations for Clinical Care 58 of Midlife Women Working Group. The North American 59 Menopause Society recommendations for clinical care of midlife 60 women. Menopause 2014;21:1038-62

84. Salthouse TA. When does age-related cognitive decline begin? Neurobiol Aging 2009;30:507-14 
Supplementary material for Lehert P. et al. Individually modifiable risk factors to ameliorate cognitive aging: a systematic review 58 and meta-analysis, Climacteric. 2015; doi: 10.3109/13697137.2015.1078106. 59

4
Supplementary Table S1 Search terms used in PubMed searches

Search category Search terms

Outcomes “memory”[MeSH Terms] OR “memory”[All Fields] OR “cognition”[MeSH Terms] OR “cognition”[All Fields] OR “cognitive”[All Fields]

Limitations

Interventions"

B vitamins (Vitamin B12, folic acid, vitamin B6) $(79 \mid 4)$

\section{DHEA $(38 \mid 1)$}

Ginkgo biloba $\left(108 \mid 0^{\dagger}\right)$

Mediterranean diet $\left(7 \mid 1^{\ddagger}\right)$

Menopausal hormone therapy $\left(237 \mid 6^{\mathbb{}}\right)$

Mindfulness (306 | 3)

Omega-3 polyunsaturated fatty acids (56 | 3) "fatty acids, omega-3"[MeSH Terms] OR “omega-3”[All Fields] OR “alpha-linolenic acid" [MeSH Terms] OR “docosahexaenoic acids" [MeSH Terms] OR "eicosapentaenoic acid" [MeSH Terms] OR "linolenic”[All Fields] OR “docosahexaenoic”[All Fields] OR "eicosapentaenoic"[All Fields] OR "n 3 polyunsaturated fatty acid”[All Fields] OR "n 3 polyunsaturated fatty acids"[All Fields]

Social engagement and social support (163|1) “social”[All Fields] AND (“engagement”[All Fields] OR "participation”[All Fields] OR "activity"[All Fields])

Soy isoflavones $(22 \mid 4)$

Vitamin D $(22 \mid 1)$

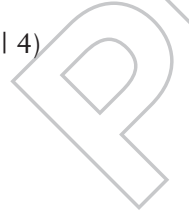

Cognitive training and cognitive activity Physical activity (aerobic exercise)
soy[All Fields] OR “isoflavones”[MeSH Terms] OR “isoflavones”[All Fields] OR

"isoflavone"[All Fields]) OR "soy foods"[MeSH Terms] OR ("soy”[All Fields] AND

"foods"[All Fields]) OR "soy foods"[All Fields] OR "tofu”[All Fields]

"vitamin d" [MeSH Terms] OR "vitamin d"[All Fields] OR "ergocalciferols" [MeSH Terms] OR "ergocalciferols"[All Fields] OR "cholecalciferol”[MeSH Terms] OR "cholecalciferol"[All Fields]

Reviewed on basis of recent meta-analysis"

Reviewed on basis of recent meta-analysis"

"Numbers in parentheses represent number of citations I number of eligible trials. ${ }^{\dagger}$ Data from one otherwise eligible trial were not in a form 106 that could be extracted for analysis (see text). ${ }^{\ddagger}$ Three publications; one trial. ${ }^{\S}$ Eight publications; six trials. An additional trial published after our systematic search is described in the text. "We did not undertake an independent systematic search on these topics (see text) 
Supplementary Table S2 Cognitive effects of individually modifiable interventions: random-effects model

\begin{tabular}{lcrrrr}
\hline & \multicolumn{2}{c}{ Global cognition } & & \multicolumn{2}{c}{ Episodic memory } \\
\cline { 2 - 3 } \cline { 5 - 6 } Intervention & $\begin{array}{c}\text { Standardized mean } \\
\text { difference }\end{array}$ & $95 \%$ CI & & $\begin{array}{c}\text { Standardized } \\
\text { mean difference }\end{array}$ & $95 \%$ CI \\
\hline B-vitamins & 0.02 & -0.01 to 0.05 & & 0.04 & 0.00 to 0.08 \\
Dehydroepiandrosterone & 0.06 & -0.10 to 0.22 & & 0.12 & -0.15 to 0.39 \\
Mediterranean diet + mixed nuts & 0.08 & 0.03 to 0.14 & & 0.07 & -0.03 to 0.17 \\
Mediterranean diet + olive oil & 0.22 & 0.16 to 0.27 & & 0.22 & 0.12 to 0.32 \\
Menopausal hormone therapy & -0.03 & -0.05 to 0.00 & & -0.01 & -0.04 to 0.03 \\
Omega-3 fatty acids & -0.02 & -0.04 to 0.01 & & 0.02 & -0.04 to 0.07 \\
Social engagement & 0.12 & -0.02 to 0.25 & & 0.20 & -0.08 to 0.47 \\
Soy isoflavones & 0.04 & 0.00 to 0.08 & & 0.11 & 0.04 to 0.17 \\
Tai chi exercise & 0.18 & 0.06 to 0.29 & & 0.24 & -0.03 to 0.51 \\
Vitamin D & 0.00 & -0.04 to 0.04 & -0.01 & -0.08 to 0.06 \\
Yoga & 0.02 & -0.12 to 0.17 & & 0.06 & -0.38 to 0.50 \\
\hline
\end{tabular}

Standard mean differences and 95\% confidence intervals by intervention for primary (global cognition) and secondary (episodic memory) outcomes. Estimates from fixed-effects and random-effects models are very similar

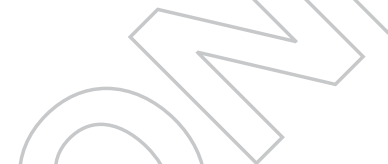

Supplementary Table S3 Effects of individually modifiable interventions: general intelligence (non-memory) outcomes

\begin{tabular}{|c|c|c|c|c|}
\hline \multirow[b]{2}{*}{ Intervention } & \multicolumn{2}{|c|}{ Fixed effects model } & \multicolumn{2}{|c|}{ Random effects model } \\
\hline & $\begin{array}{l}\text { Standardized } \\
\text { mean difference }\end{array}$ & $95 \% \mathrm{CI}$ & $\begin{array}{l}\text { Standardized } \\
\text { mean difference }\end{array}$ & $95 \% C I$ \\
\hline B-vitamins & 0.01 & -0.03 to 0.04 & 0.00 & -0.04 to 0.04 \\
\hline Dehydroepiandrosterone & 0.09 & -0.13 to 0.31 & 0.09 & -0.14 to 0.31 \\
\hline Mediterranean diet + mi & ค & 0.00 to 0.15 & 0.08 & 0.00 to 0.15 \\
\hline Mediterranean diet + & 0.21 & 0.14 to 0.28 & 0.21 & 0.14 to 0.29 \\
\hline Menopausal hormone the & -0.03 & -0.06 to 0.01 & -0.02 & -0.06 to 0.02 \\
\hline Omega-3 fatty acids & -0.04 & -0.08 to -0.01 & -0.05 & -0.09 to 0.00 \\
\hline Social engagement & 0.09 & -0.06 to 0.24 & 0.09 & -0.06 to 0.24 \\
\hline Soy isoflavon & 0.01 & -0.05 to 0.06 & 0.01 & -0.05 to 0.06 \\
\hline Tai chi exercis & 0.14 & 0.01 to 0.27 & 0.14 & 0.01 to 0.27 \\
\hline Vitamin $/$ & 0.00 & -0.06 to 0.06 & 0.00 & -0.07 to 0.07 \\
\hline Yoga & 0.02 & -0.13 to 0.17 & 0.02 & -0.14 to 0.17 \\
\hline
\end{tabular}

Standard mean differences and $95 \%$ confidence intervals by intervention for general intelligence outcomes. General intelligence was not a primary or secondary outcome, and results are not interpreted in the text 